\title{
Evaluating Urban Community Sustainability by Integrating Housing, Ecosystem Services, and Landscape Configuration
}

\author{
Liang Zhou, ${ }^{1,2}$ Haowei Mu $\mathbb{D}^{1},{ }^{1,3}$ Bao Wang, ${ }^{4}$ Bo Yuan $\mathbb{D D}^{1,3}$ and Xuewei Dang ${ }^{1,5}$ \\ ${ }^{1}$ Faculty of Geomatics, Lanzhou Jiaotong University, Lanzhou 730070, China \\ ${ }^{2}$ State Key Laboratory of Resources and Environmental Information System, \\ Institute of Geographic Sciences and Natural Resources Research, CAS, Beijing 100101, China \\ ${ }^{3}$ National-Local Joint Engineering Research Center of Technologies and Applications for National Geographic State Monitoring, \\ Lanzhou 730070, China \\ ${ }^{4}$ Northwest Institute of Eco-Environment and Resources, CAS, Lanzhou 730000, China \\ ${ }^{5}$ Gansu Provincial Engineering Laboratory for National Geographic State Monitoring, Lanzhou 730070, China
}

Correspondence should be addressed to Haowei Mu; 0218742@stu.lzjtu.edu.cn and Bo Yuan; 0219795@stu.lzjtu.edu.cn

Received 6 July 2020; Revised 11 August 2020; Accepted 12 August 2020; Published 27 August 2020

Academic Editor: Jun Yang

Copyright (c) 2020 Liang Zhou et al. This is an open access article distributed under the Creative Commons Attribution License, which permits unrestricted use, distribution, and reproduction in any medium, provided the original work is properly cited.

\begin{abstract}
Community is the core spatial unit for evaluating sustainable development. However, single data and method seem inadequate for conducting a scientific, effective, and innovative sustainable evaluation of complex community units. In this study, we perform a sustainable-oriented land use scheme using multisource remote sensing, machine learning, and object-based postclassification refinement. Furthermore, we assess the sustainability of the traffic community by data-driven and combined housing, ecosystem services, and landscape configuration. The results indicated that (1) the relationship between housing, ecosystem services, and landscape pattern has obvious synergistic effects, although with dissimilar importance in different sustainability levels. High sustainability level is intensely coordinated with landscape configuration, medium sustainability level is more affected by ecosystem services, and low sustainability level is more related to housing. (2) Community sustainability presents a significant spatial distribution. The communities of high sustainability level are mainly located in both sides of the Pearl River and emerging urban areas, while those of medium sustainability level are distributed sporadically in the study area and those of low sustainability level are concentrated in old towns. (3) Community transformation cannot be accomplished at one step. Along with the continuous optimization of landscape configuration, the priority should be given to housing reconstruction and improvement of ecosystem services further. We provide scientific and effective data-based evidence for urban decision-makers by integrating the advantages of the Earth Observation System and multifactor analysis.
\end{abstract}

\section{Introduction}

Cities are hubs for ideas, science, culture, commerce, productivity, social development, etc. [1,2], which were deemed as sustainability problems rather than solutions in the last few decades $[3,4]$. Nowadays, cities have become a core to ensuring a sustainable future and human well-being $[5,6]$. Some of the largest economies in the world, such as the US, the UK, and Japan, have already developed their sustainable community rating framework while lacking in China $[7,8]$. Rapid urbanization has caused increasing social-environmental problems, such as uneven income, unfair housing cities poverty, and environmental deterioration, which exhibit a substantial negative impact on urban and regional sustainable development $[9,10]$. Therefore, it urgently needs to launch a sustainable community evaluation system in China.

Currently, the evaluation of sustainable community relies typically on the construction industry $[11,12]$, and more industries such as information and communication, and energy are joining in the sustainable assessment of urban communities [13]. As building sustainable cities and communities was defined as one of the sustainable development goals (SDGs) by the United Nations in the 2030 
agenda, the selection of urban development orientation will face more severe challenges, especially for developing countries [14, 15]. Additionally, SDGs 11.1 explicitly proposes informal housing and slum renovation and SDGs 11.3 proposes to enhance the planning and management capacity of sustainable human settlements. A good understanding of the complexity and dynamics of sustainable urban development under different development pathways is necessary for cities to achieve SDGs $[16,17]$. Thence, it is particularly important to combine SDGs with the perspective of academia.

Academia believes that the key to urban sustainability is economics, environment, and society [18, 19]. There has been some classical and innovative work on quantifying mechanisms for translating urban elements into sustainability [20, 21]. However, utilizing the timeliness of remote sensing data to evaluate sustainability at the community scale is still rare. The different definition of urban sustainability is "adaptive processes that promote and sustain the virtual cycle between ecosystem services and human wellbeing in a coordinated manner [22]." While varying in certain aspects, the definition of urban sustainability incorporates environment, economic, and human well-being. Successful sustainable communities require consideration of additional dimensions [23]. For instance, urban planning scholars applied livability to a sustainable evaluation in urban communities [24]. Livability encompasses the overall perception of the city by its inhabitants, and from this vague perception, we can identify key issues such as compactness, housing, and ecological health. Ecosystem services contribute directly and indirectly to human well-being, and landscape design harmonizes the man-land relationship and guarantees the long-term development of mankind.

Some studies establish a comprehensive indicator system by statistics data to evaluate sustainability in communities $[25,26]$. However, community sustainability evaluation must flexibly and timely adapt to the city's distinguishing feature $[8,27]$. With the establishment of the Earth $\mathrm{Ob}-$ servation System (EOS), which has a finer spatial resolution than statistical data, and the development of machine learning, flexibly, and timely evaluating sustainability in communities has transformed from an idea into a reality [28]. The EOS is conducive to information mining and expressions, such as mapping informal settlements [29], urban phenology, and heat island [30,31], and quantifying environmental quality and risk $[32,33]$. Therefore, we can find a new path to achieve sustainability evaluation by EOS from the perspective of housing, ecosystem services, and landscape configuration in communities. Among them, housing, which includes storied buildings, informal housing, and villas, represents different economic and social strata. The ecosystem services and landscape configuration represent different ecological and environments. These indicators meet the requirements of urban sustainability evaluation. Land use and land cover classification, which provides a potential basis for sustainable evaluation, is an ordinary task in EOS. The ecosystem service values (ESVs) can be calculated with a clear, operational mechanism by land cover, and sustainability indicators such as housing and landscape configuration can be transformed by land use in urban communities [34, 35]. To this end, combined with the concept of sustainable development, we designed an efficient, cloud-based, and data-driven evaluation framework. Sustainable evaluation is typically related to the value judgment of people [36]. We classify levels of community sustainability by combine data and value judgment of people.

The achievement of SDGs needs science, technology, and innovation. It is indispensable to integrate Earth observation data and machine learning methods to develop a data-driven sustainable evaluation scheme. The study was based on fusing optical and SAR images and machine learning methods by OBPR to classify urban land cover into eight categories, especially storied buildings, informal housing, and villas (Section 4.1). Then, it converts this land use and land cover into housing, ESV (Section 4.2), and landscape configuration (Section 4.3) according to the connotation of sustainability. Finally, we obtained three rating levels to evaluate sustainability in urban communities (Section 4.4). In summary, we made full use of remote sensing to reduce the impact of human factors and carried out a real-time, efficient, and sustainable evaluation in urban communities.

\section{Study Area and Datasets}

2.1. Study Area. Guangzhou, located at the north Pearl River Delta, is the central city of South China. As the capital of Guangdong province, Guangzhou is the center of politics, economy, science, and education in South China (see Figure 1). This study mainly talks about some subcities or districts, including Yuexiu, Tianhe, Haizhu, Liwan, and Panyu (see Figure 1(a)). At the end of 2018, the urban population accounted for $86.38 \%$ in Guangzhou, and the per capita disposable income of urban residents was $\$ 8478.76$ in each city. In May 2012, Guangzhou initiated the "multiple-plan integration" [37] to explore the future development of megacities in China. Nowadays, high-density population, economic pressures, and ecological requirements pose challenges to sustainable development in Guangzhou [38, 39].

2.2. RS Data and Sample Collections. The study selected 27 Sentinel-2 MSI spectral images from May 1st to September 20th, 2019, and operated cloud processing in the QA60 band of the data. The research data were obtained by mean synthesis after removing opaque clouds and cirrus clouds, and the preprocessing of Sentinel-1 images and SAR images were operated on the GEE platform. The preprocessing includes the following: (1) removal of thermal noise, lowintensity noise, and invalid data on scene edges; (2) radiometric calibration; (3) conversion of data from ground range geometry to backscatter coefficient using the 30-Meter SRTM or the ASTER DEM for the high latitude, and the synthesized data are resampled to a resolution of 10 meters.

Urban land use is divided into the following eight types (see Table 1), namely, storied building (SB), informal housing $(\mathrm{IH})$, villas $(\mathrm{V})$, industry $(\mathrm{I})$, bare land $(\mathrm{BL})$, trees 



Figure 1: Study area and data examples. (a) Study area. (b, c) Data examples of the traffic community. (d) Study area range. (e) Sentinel-2A optical image (true colour composition). (f) Sentinel-1A SAR image (red: VV; green: VH; blue: VV/VH).

(T), lawn and crop (LC), and water (W) (see Figure 2). Among them, SB consists of concrete buildings and regular shape, IH is made of colour steel composite panels which are easily broken, and the distinction among $\mathrm{V}$ is mainly through its characteristics and red roof. The three dwelling places represent distinct personal financial levels and social status. The samples of eight types were obtained by visual sampling on GEE while borrowing Sentinel-2 and objectoriented results.

2.3. Traffic Community. The traffic community is a scheme, which is proposed to reduce the complexity of traffic control and management systems to improve system reliability and system development. The traffic community is obtained under three principles: (1) homogeneity-land use, economy, society, and other characteristics within the communities are as consistent as possible; (2) partition boundary is natural barriers (high-grade roads, railways, and rivers); (3) the areas of the city center are smaller, while the suburbs are larger. In this study, traffic community data are derived from the China Academy of Urban Planning and Design (http:// www.caupd.com).

\section{Methods}

The framework of sustainability evaluation in urban communities is presented in Figure 3. First, we have classified land use and land cover by fused on optical and SAR employ machine learning in GEE (Figure 2(a)). A sustainable- 
TABLe 1: Statistics of training and test samples at each land use class.

\begin{tabular}{lccc}
\hline Class & Training samples & Test samples & Total \\
\hline Storied building (SB) & 172 & 68 & 240 \\
Informal housing (IH) & 216 & 93 & 309 \\
Villas (V) & 179 & 60 & 239 \\
Industry (I) & 117 & 56 & 173 \\
Bare land (BL) & 47 & 25 & 72 \\
Trees (T) & 148 & 56 & 204 \\
Lawn and crop (LC) & 144 & 66 & 210 \\
Water (W) & 86 & 34 & 120 \\
Total & 1109 & 458 & 1567 \\
\hline
\end{tabular}

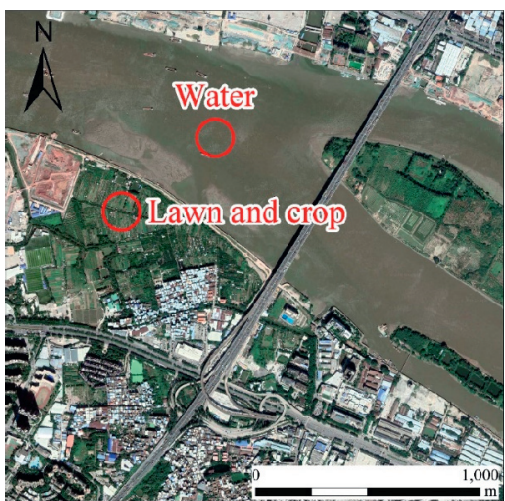

(a)

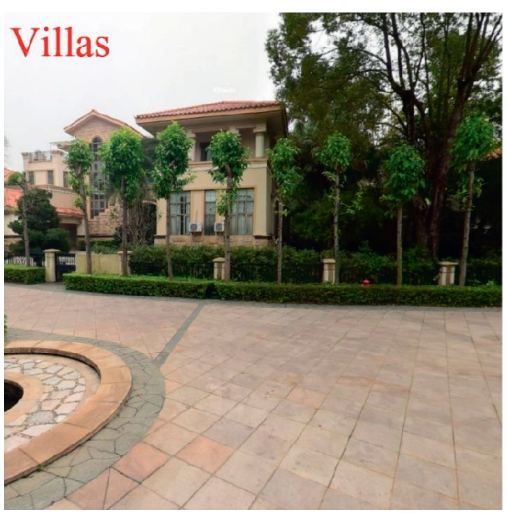

(d)

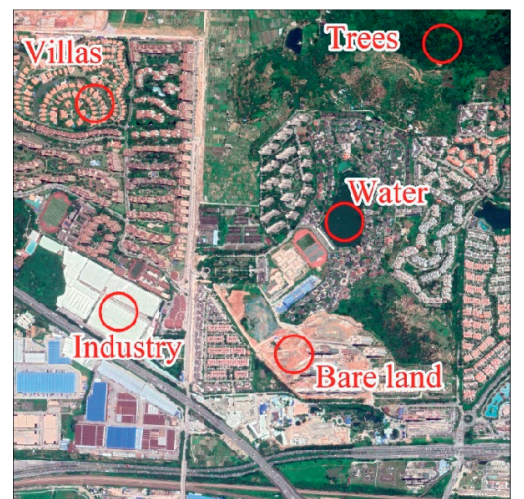

(b)

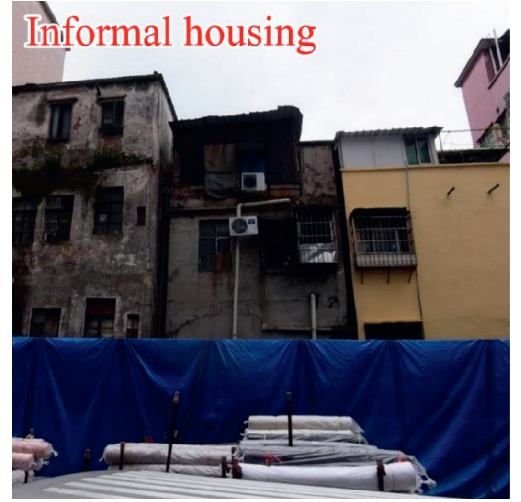

(e)



(c)



(f)

FIgURE 2: Typical land use categories in the study area.

oriented land use scheme has been built. Then, we transform land use and land cover into housing, ESV, and landscape configuration. Finally, we divide three levels of community sustainability using $k$-means by a data-driven method (Figure 2(b)).

3.1. Support Vector Machine and Random Forest. Support vector machine (SVM) [40] is a kind of supervised learning with associated learning algorithms. In the case of linear inseparability, SVM first completes the calculation in lowdimensional space and maps the input space to the high- dimensional feature space than through the kernel function. Finally, the optimal separating hyperplane is constructed in the high-dimensional feature space. This study uses the radial basis function kernel, which is a prevalent kernel function in machine learning.

Random forest (RF) [41] is a method for discriminating and classifying data using multiple classification trees. The method randomizes the row and column data selections, generates some classification trees, and summarizes the results of the classification tree then. RF improves prediction accuracy without significantly increasing the amount of computation. Its results are robust to lost and unbalanced 


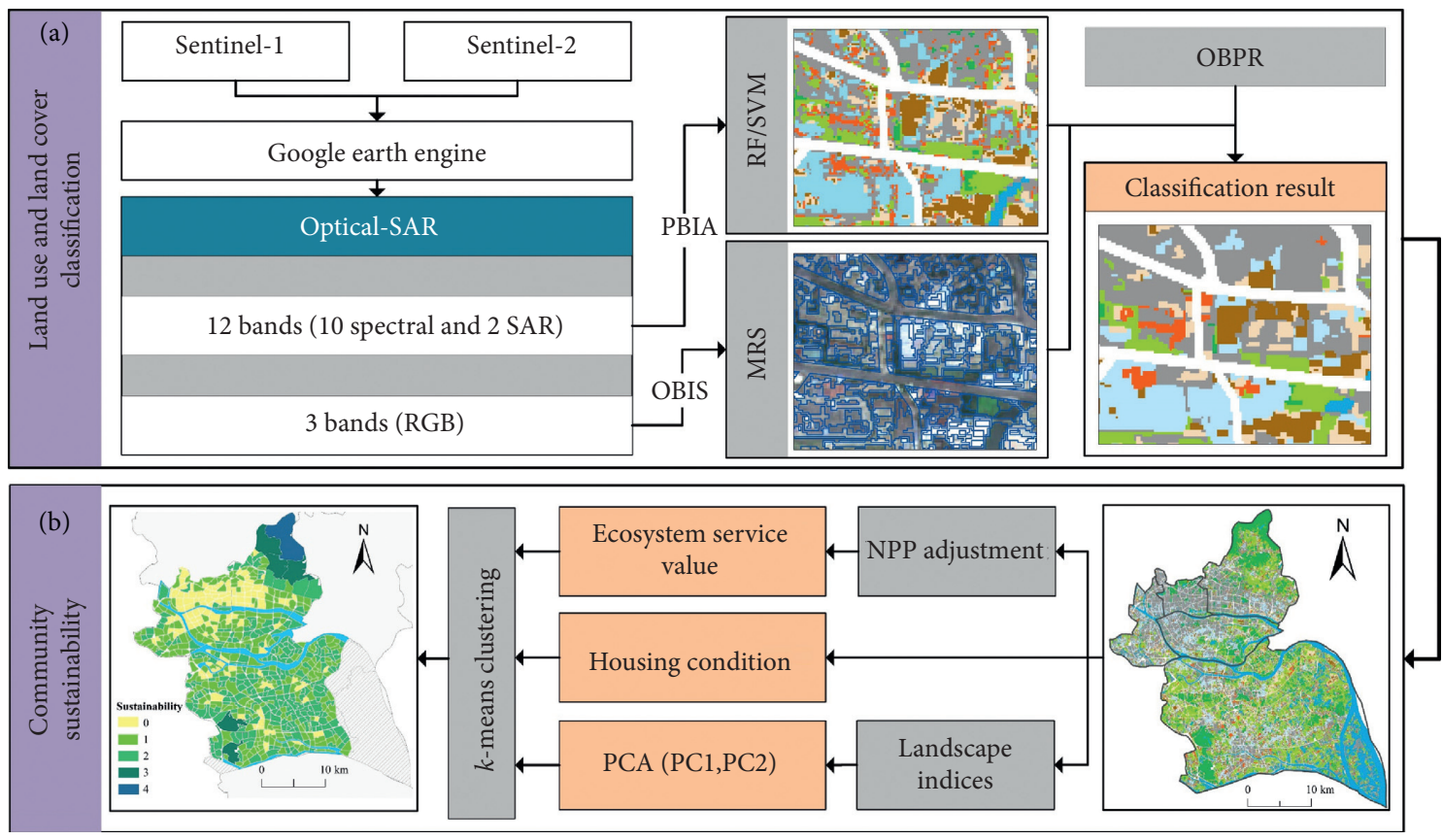

FiguRE 3: The framework of sustainability evaluation in urban communities.

data and can well predict the effects of up to several thousand explanatory variables, which are still widely used today $[42,43]$.

3.2. OBPR. OBPR [44] is employed after pixel-based image analysis (PBIA) and object-based image segmentation (OBIS). After the pixel classification, each pixel $x$ gets a classification result $T$, which is the type of land use classification. On the assumption of correct OBIS results, the pixels in the same object $O$ should be the same classification type, where $O=\left\{\left(x_{1}, T_{1}\right),\left(x_{2}, T_{2}\right), \ldots,\left(x_{k}, T_{k}\right)\right\}, k$ is the number of pixels in $O$, assuming $f\left(T_{k}\right)$ is the ratio of $T_{k}$ in the object patch $O$, and then the predicted object type $O$ can be calculated as

$$
\begin{aligned}
& O=\arg \max \left(f\left(T_{k}\right)\right), \\
& R=\left(1-\max \left(f\left(T_{k}\right)\right)\right) \times 100 .
\end{aligned}
$$

$R$ represents the classification risk of the dominant category in the object.

3.3. Ecosystem Service. Ecosystem services contribute to human well-being, both directly and indirectly, and represent part of the total economic value of the planet $[45,46]$. The ESV method acquired adaptive improvement to fit situations in China [47]. The basic equivalent of ESV function per unit area refers to the annual average value equivalent of various service functions per unit area of different types of ecosystems. ESV was calculated through T and LC in this study. Considering the complex urban system and associating with the previous research results, the ecosystem service functions were divided into regulating services, support services, and cultural services. The basis weight and the net primary production (NPP) spatiotemporal adjustment factor were designed with reference to the evaluation of ESV by Xie in this study. Annual NPP is derived from the sum of all 8-day net photosynthesis (PSN) products (MOD17A2H) from the given year [48]. Finally, the adjusted NPP spatiotemporal adjustment factor is calculated as

$$
p_{i j}=\frac{B_{i j}}{\bar{B}},
$$

where $B_{i j}$ is the PSN sum in month $j$ within the ecosystem region $i$ and $\bar{B}$ represents the annual average PSN of each ecosystem nationwide.

We calculated $p_{i j}$ with MODIS data, where Guangzhou was used as a spatial reference, and the time span of spectral data was used as a time reference. Thus, the equivalent coefficient of ESV per unit area was revised (see Table 2). The product of the equivalent coefficient and the corresponding land cover areas is the ESV for different ecological service functions.

3.4. Landscape Indices. According to the experimental needs, we select nine landscape indicators to express the overall structural characteristics of the traffic community, which are the number of patches (NP), patch density (PD), largest patch index (LPI), landscape shape index (LSI), contagion (CONTAG), landscape division index (LDI), splitting index (SI), Shannon's diversity index (SHDI), and Shannon's evenness index (SHEI). The fact that the landscape indices are not completely independent of each other, which will result in a repetitive description of the information, whilst some researchers point out that the correlation of the landscape indices does not indicate that an index ought to be eliminated $[18,49]$. Accordingly, principal component analysis (PCA) was used to select two principal 
TABLE 2: The equivalent coefficient of ESV per unit area after time-space adjustment (yuan).

\begin{tabular}{lcccccc}
\hline \multirow{2}{*}{ Ecosystem category } & \multicolumn{2}{c}{ Regulating } & \multicolumn{2}{c}{ Supporting } & \multicolumn{2}{c}{ Cultural } \\
& Gas & Climate & Purification & Nutrient cycle & Biodiversity & Aesthetics \\
\hline T & 2.21 & 6.61 & 1.96 & 0.20 & 2.45 & 1.08 \\
LC & 1.95 & 4.63 & 1.52 & 0.20 & 1.93 & 0.85 \\
\hline
\end{tabular}

components of nine landscape indicators in this study. PCA is one of the most useful methods for studying urban sustainability indicators [50], and cumulative contributions in each component beyond $80 \%$ are favorable for sustainability assessment.

\section{Results}

4.1. House-Oriented Land Use Classification Results. The object patch is segmented from the optical and SAR images using eCognition's multiresolution segmentation (MRS) algorithm. Based on visual interpretation, the appropriate scale parameter is set to 30 , the tightness parameter is 0.8 , and the shape parameter is 0.1 . After MRS completed, the traffic community was used to cut MRS results apart into 188,437 object patches. Finally, we used the OBPR method which combined PBIA and OBIS results to obtain the final land use classification then.

The classification results are shown in Table 3, and the accuracy of SVM and RF on eight land use types improved significantly after using OBPR. For OBPR-RF results, the accuracy of $\mathrm{I}$ and $\mathrm{W}$ increases to $100 \%$, while $\mathrm{T}$ accuracy reduces and $\mathrm{SB}$ and $\mathrm{BL}$ remain unchanged. The OBPR-RF achieves a higher classification accuracy (OA is $92.79 \%$, and $k$ is 0.92 ) than $\mathrm{RF}(\mathrm{OA}$ is $90.17 \%$, and $k$ is 0.88 ). For OBPRSVM results, the accuracy of $\mathrm{W}$ increases to $100 \%$, while the accuracy of $\mathrm{V}$ decreases, and I remains unchanged.

After OBPR, most ground objects are well classified. The classification results of SB and $\mathrm{IH}$ have reached the experimental requirements with appearing in the form of a contiguous area, which is in line with actual surface features (e.g., in Figures 4(a2) and 4(b2)). Dense ground objects blend various pixels, which contains more land use categories but difficultly classifies in machine learning. The risk map (e.g., in Figures 5(a) and 5(b)) shows that the classification risks of $\mathrm{W}, \mathrm{T}$, and $\mathrm{SB}$ are low, and $\mathrm{IH}$ is high, which because $\mathrm{W}, \mathrm{T}$, and SB are all continuous and homogeneous, and the difference in spectral features is small. Simultaneously, the map indicates that the more complex ground object accompanied with the higher classification risk.

4.2. ESV for Community. In this study, the ESV shows differences in traffic communities (see Figure 5(a)). We talk about the ratio of different land use types of $\mathrm{IH}$ (see Figure 5(b)), SB (see Figure 5(c)), and V (see Figure 5(d)) in the traffic community to assess urban ecological sustainability. We excluded the farmland-based traffic community because it contributes little significance to urban sustainability. The results show the obvious differences exist in ESV of the dominant land use types. The lower ESV in the traffic community with a higher ratio of SB. The IH mostly distributes in areas with low ESV. V has a high proportion beside the river and mainly distributed in high ESV areas. This fact is consistent with the general ideas of municipal developers, which proves that the quality of the urban social environments is strongly related to socioeconomic status in developed regions $[51,52]$. This definite spatial distribution and quantification methods are beneficial to urban planning and sustainable development, whilst urban renewal in units of traffic communities may be a good option.

The average ratio of land use types in the traffic communities of different districts is shown in Figure 6. Liwan, Yuexiu, and Haizhu are older towns in Guangzhou. The proportion of SB in Yuexiu has reached 70\%, approximately one million people live here, and trees have a significant contribution to regulate ecosystem services. Liwan holds a higher proportion of SB and IH than other older towns, and the proportion of urban green space is small and dominated by grassland. Although the ratio of $\mathrm{IH}$ is the highest in Haizhu, it shows a high ESV brought by the surrounding river. Considering that these older towns are mainly residential places, IH transformation, and ecosystem service enhancement are in the focus field of urban renewal, and it is the inevitable problem faced by people-oriented urban sustainable development. Panyu is one of the top investment potential areas in Guangzhou. LC is mainly cropland and less diverse than $\mathrm{T}$, and considerable land will be transformed into SB and V in the future. Tianhe is regarded as a representative of commercial prosperity in Guangzhou, and SB is mainly commercial places. Built on land use results, it can be found that urban green spaces are dominated by trees, a positive relationship between plant diversity and human wealth in evidence [53].

4.3. Landscape Configuration for Community. PCA was carried out after the normalizing of the landscape index in each traffic area, with the cumulative contribution rate of PC1 and PC2 reaching 81\% (see Table 4), of which CONTAG, LPI, SPLIT, SHDI, SHEI, and DIVISION mainly contribute to PC1, NP, and LSI mainly contribute to PC2 (see Figure 7). Among them, Figure 7(a) shows that positive correlations exist between three landscape indices and two principal components, respectively, in the first quadrant. The two landscape indices in the second quadrant are negatively correlated with PC1while a positive correlation with PC2, and the four landscape indices in the fourth quadrant are positively correlated with PC1 while a negative correlation with PC2. LSI contributes the most to PCA, and PD contributes the least to PCA. A higher correlation exists between indices that contribute more to the same principal component (see Figure 7(b)). There were some correlations between the original landscape indicators, such as NP and 
TABLE 3: Results of classification accuracy (\%) of different methods.

\begin{tabular}{lcccccc}
\hline Classification accuracy & SVM & RF & SVM & OBPR-SVM & RF & OBPR-RF \\
\hline Storied building (SB) & & Train & & & & Validation \\
Informal housing (IH) & 79.65 & 99.42 & 83.82 & 94.12 & 94.12 & 94.12 \\
Villas (V) & 85.65 & 99.07 & 79.57 & 83.87 & 76.34 & 83.87 \\
Industry (I) & 96.30 & 98.32 & 90.00 & 81.67 & 88.33 & 93.33 \\
Trees (T) & 97.97 & 100.00 & 96.43 & 96.43 & 98.21 & 100.00 \\
Bare land (BL) & 87.23 & 97.00 & 94.64 & 98.21 & 96.43 & 91.07 \\
Lawn and crop (LC) & 70.14 & 100.00 & 74.00 & 88.00 & 88.00 & 88.00 \\
Water (W) & 98.84 & 100.00 & 97.06 & 78.79 & 92.42 & 96.97 \\
Overall accuracy (OA, \%) & 84.76 & 99.37 & 86.24 & 89.00 & 97.06 & 100.00 \\
Average accuracy (AA, \%) & 86.30 & 99.34 & 87.47 & 90.14 & 90.17 & 92.79 \\
Kappa coefficient ( $k$ ) & 0.82 & 0.99 & 0.84 & 0.87 & 91.37 & 93.42 \\
\hline
\end{tabular}
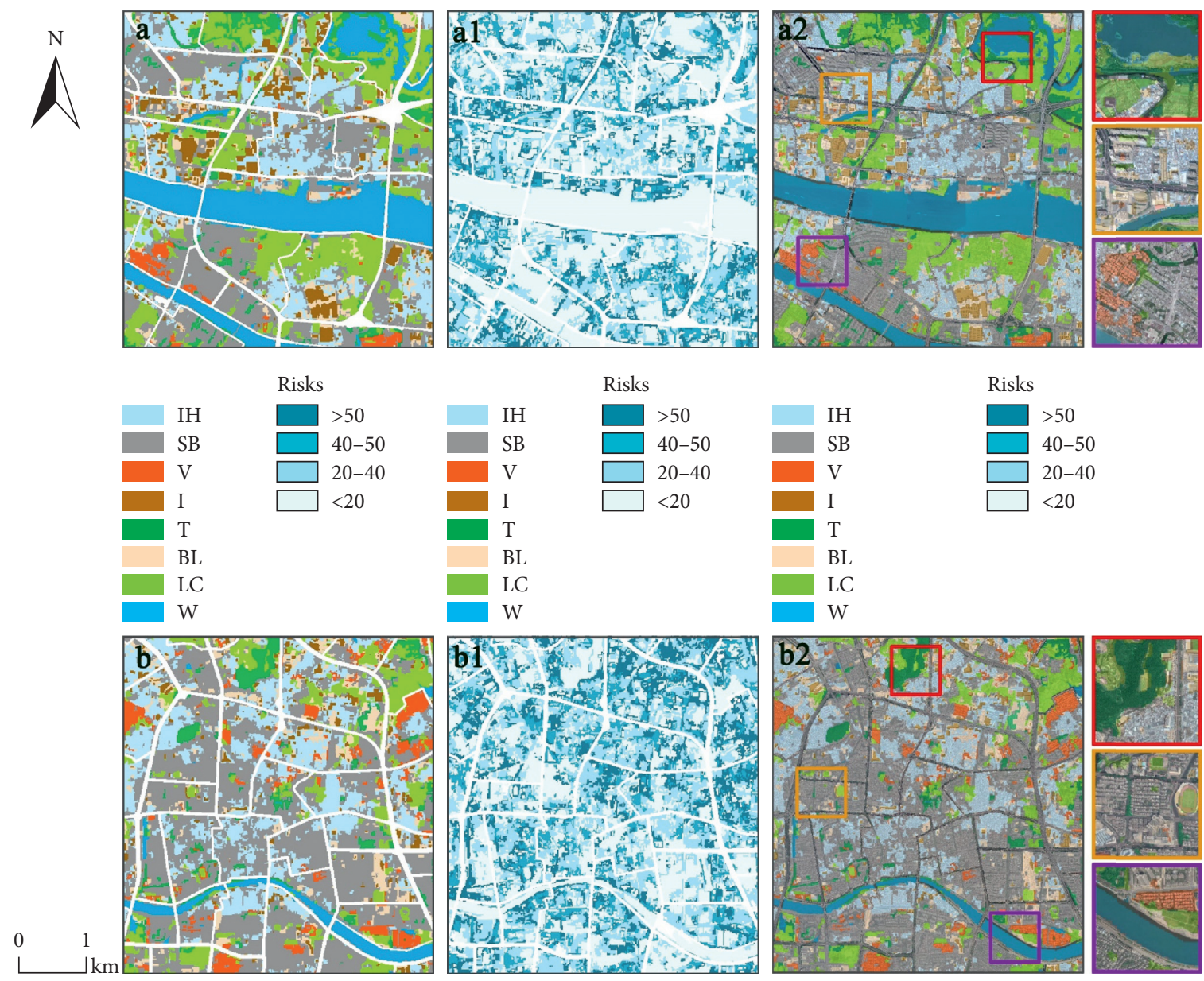
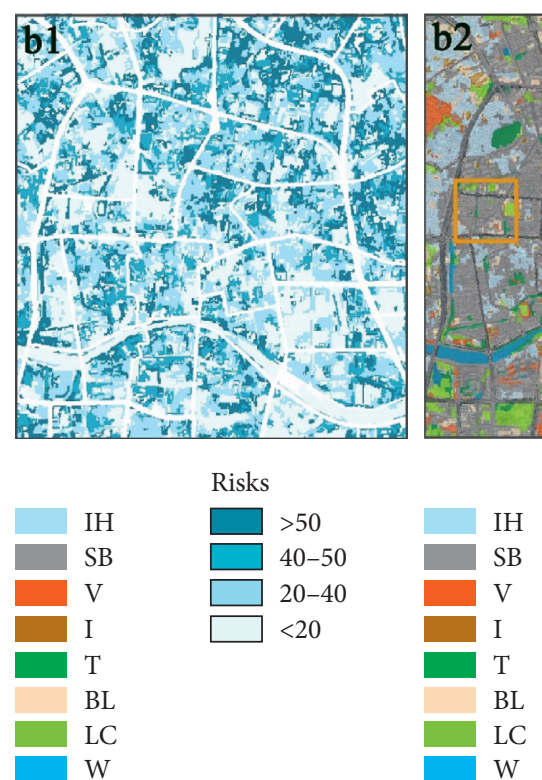

Risks

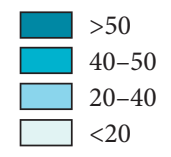

$<20$
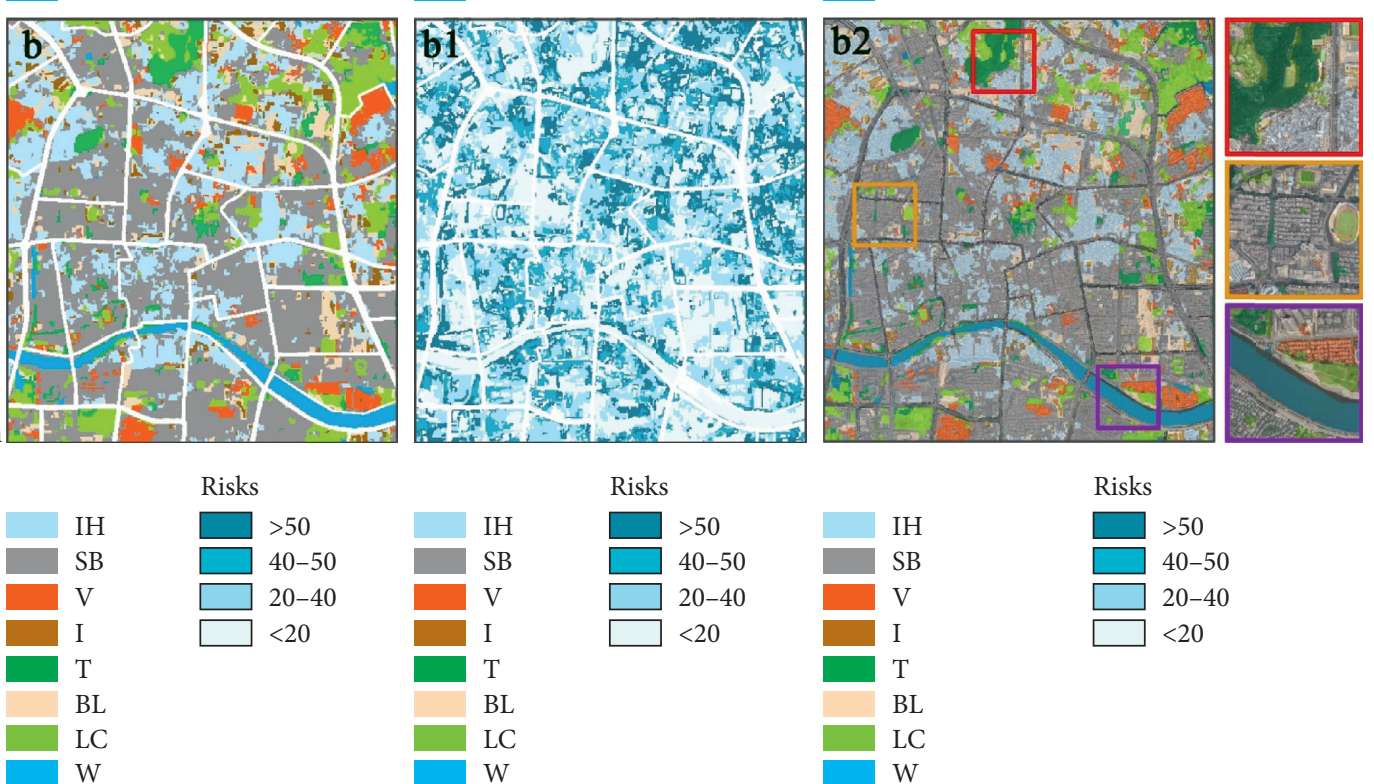

FIGURE 4: Details of urban land use classification. (a-d) Classification result examples. (b-e) The risk map of classification results. (c-f) Details of classification overlaying on Google Maps. 


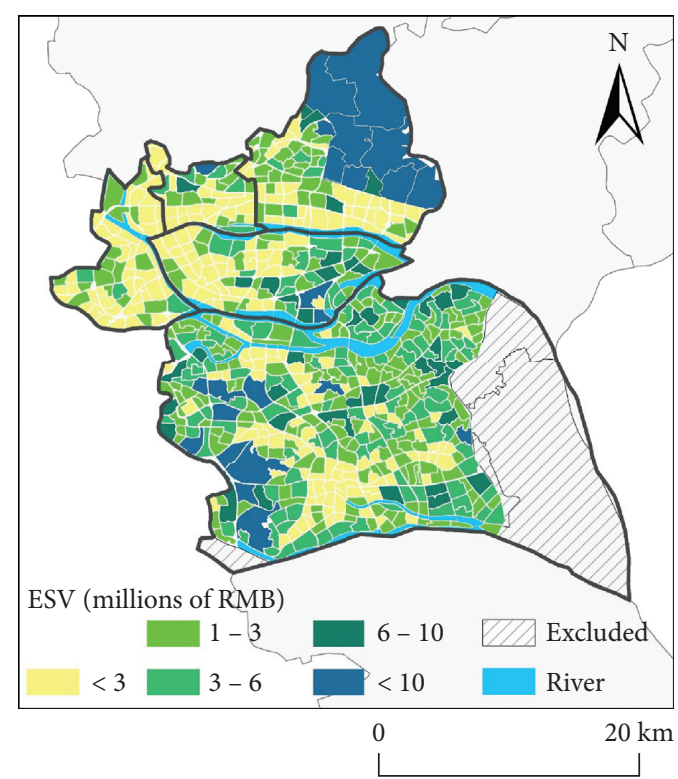

(a)

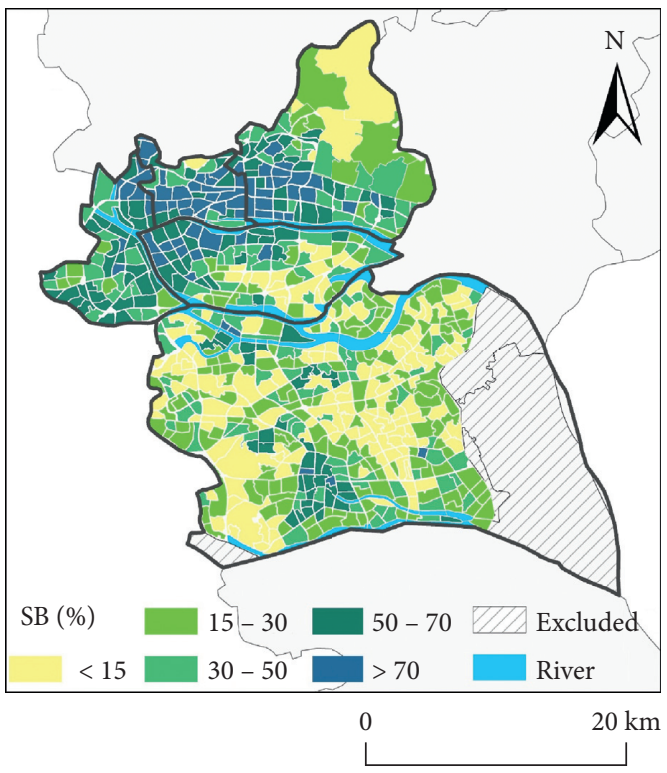

(c)



(b)

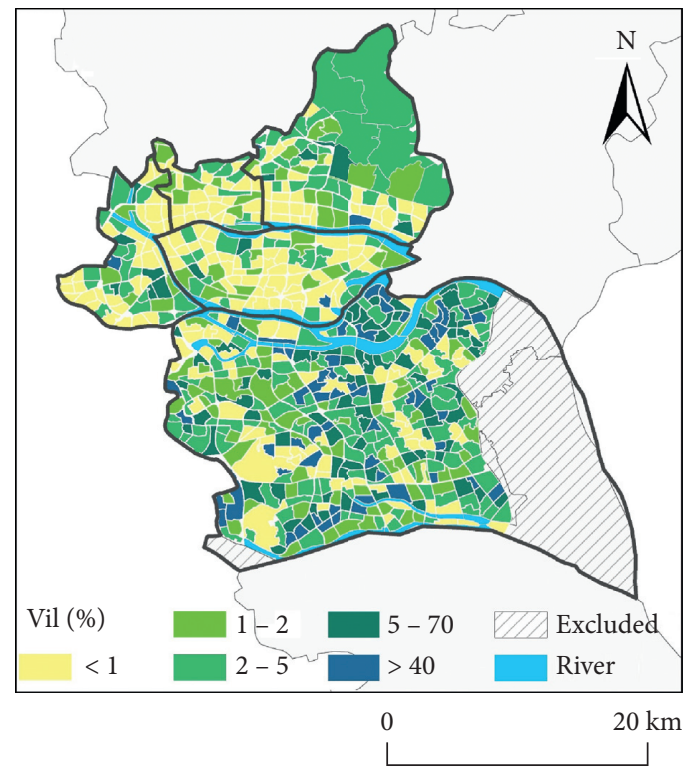

(d)

FIgURE 5: The ESV and the proportion of three different land use types in the traffic community. (a) ESV. (b) Informal housing. (c) Storied building. (d) Villas.

LSI, LPI and CONTAG, DIVISION, SPLIT, SHDI, and SHEI, as well as high correlations between the four indicators and high contributions to PC1. Therefore, we consider the principal component of PCA as a more comprehensive index to evaluate the sustainability of the landscape.

Landscape sustainability is a key approach to achieving sustainable development goals [54]. Integrating the contribution of different landscape indices to the principal components increases statistical and landscape significance. The results (see Figure 8) indicate that in the areas with a small value of PC1, the landscape diversity is low, the land use information is not rich, the patches appear contiguous, and dominant patches are forming good connectivity.
Referring to land use and requirement of landscape sustainability, it is found that these areas are mainly distributed on the SB, where the value of ecosystem services is low, and the sustainability is weak. For the area with high PC1 value, the patch types are evenly distributed with significant diversity and abundant land use information. Meanwhile, many small blocks exist in the landscape, the degree of landscape configuration aggregation is low, and the degree of separation between patches is high. For PC2, the result is different from that of PC1. This is because the landscape index giving the dominant contribution is different, in which indices are calculated by the same traffic community. The larger patch has an impact on the calculation of the 


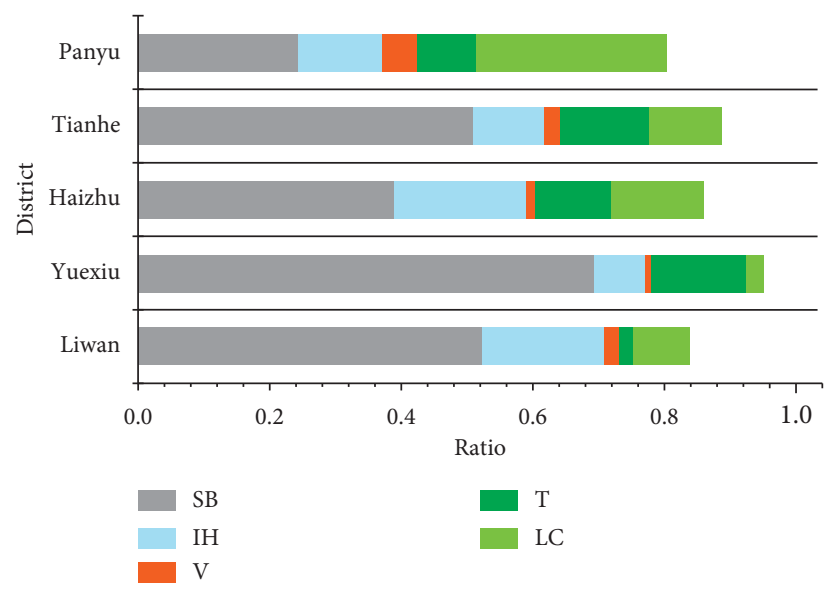

Figure 6: The average ratio of five land use types in study districts.

TABle 4: Information and proportion of each component.

\begin{tabular}{lcccc}
\hline Component & Information & Difference & Proportion & Cumulative \\
\hline PC1 & 5.7516 & 4.1484 & 0.6391 & 0.1781 \\
PC2 & 1.6033 & 0.8563 & 0.0830 & 0.8172 \\
PC3 & 0.7470 & 0.3146 & 0.0480 & 0.9002 \\
PC4 & 0.4324 & 0.1964 & 0.0262 & 0.9483 \\
PC5 & 0.2360 & 0.1156 & 0.0134 & 0.9745 \\
PC6 & 0.1204 & 0.0409 & 0.0088 & 0.9878 \\
PC7 & 0.0795 & 0.0612 & 0.0020 & 0.9967 \\
PC8 & 0.0184 & 0.0068 & 0.0013 & 0.9987 \\
PC9 & 0.0115 & 0.0000 & & 1.0000 \\
\hline
\end{tabular}

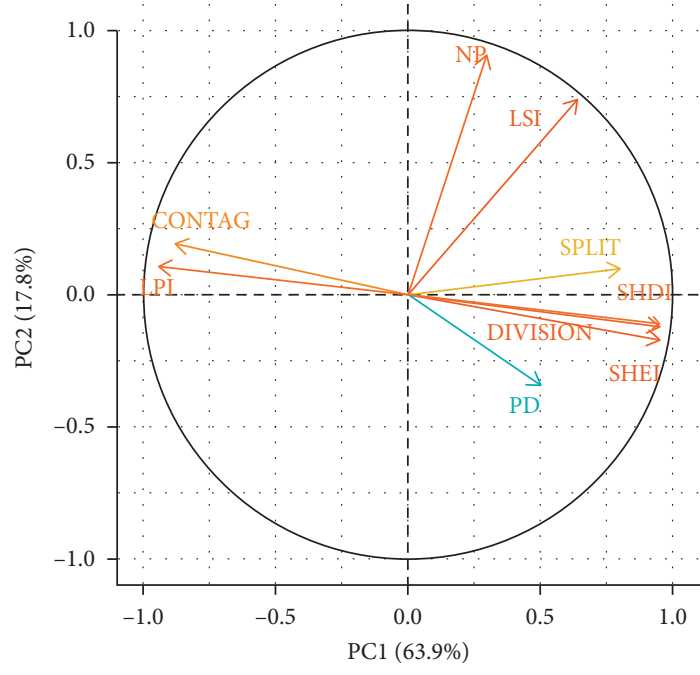

(a)



(b)

FiguRE 7: The composition contribution rate and related coefficient matrix of each landscape index. (a) Contribution of the various landscape indices to PC1 and PC2. (b) Correlation coefficient matrix for each landscape index.

landscape index. Therefore, when using PCA for landscape sustainability analysis in subsequent studies, it should be on a similar patch scale.
4.4. Community Sustainability Evaluation. We use the $k$ means clustering algorithm based on ecosystem service and landscape indices to assess urban sustainability. The code 


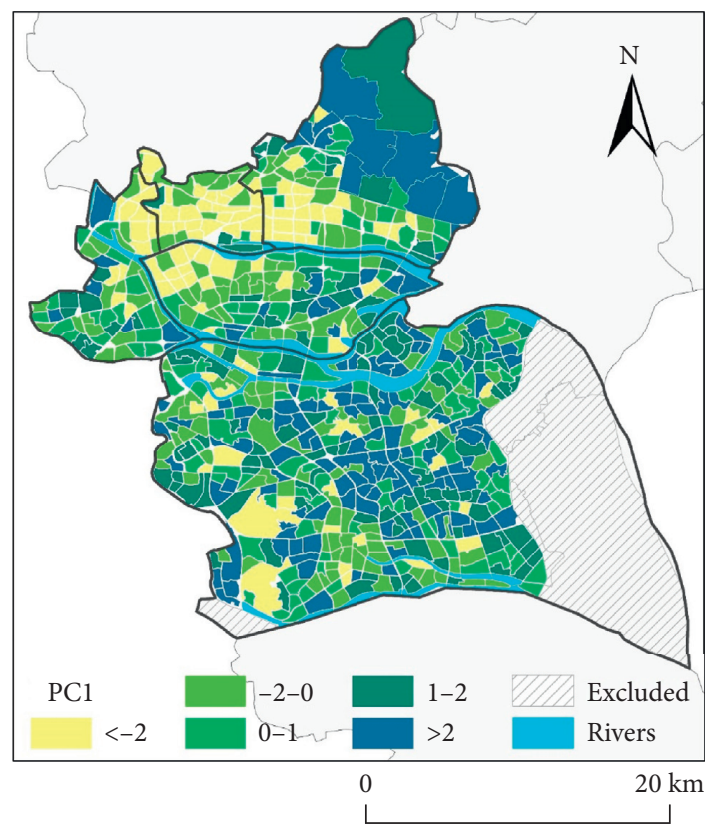

(a)

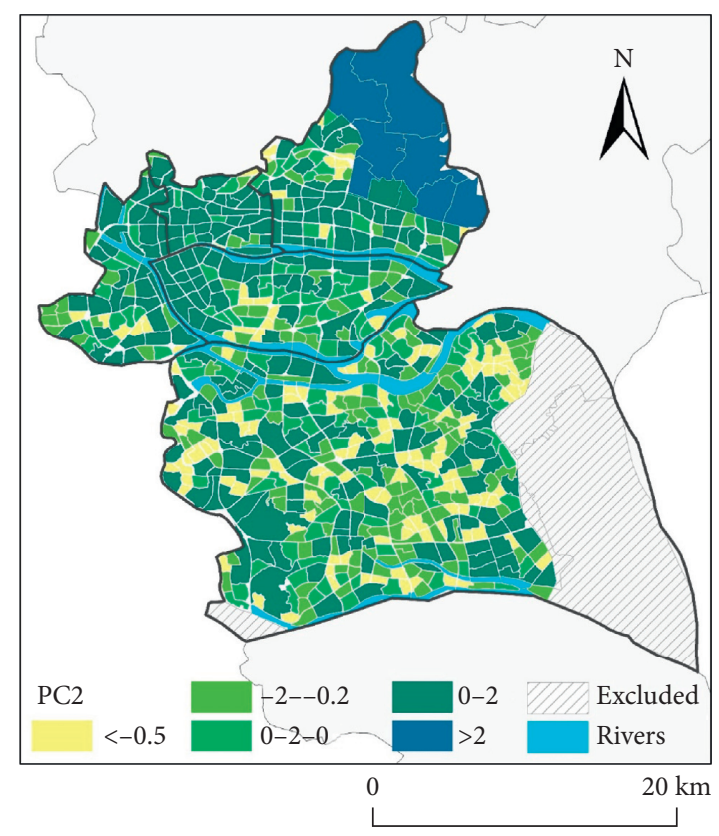

(b)

Figure 8: The distribution of PC1and PC2 values in the traffic community. (a) PC1. (b) PC2.

with the Scikit-learn library is executed on Python 3.6, which sets five centroids, and the initialization method is $k$-means ++ , the maximum number of iterations of the $k$-means algorithm is 500 , the random number initialized by the centroid is 0 , and the number of times of running under different centroid seed is 3 . The input data involved in the calculation are $\mathrm{PC} 1, \mathrm{PC} 2, \mathrm{ESV}, \mathrm{SB}, \mathrm{IH}$, and V. According to these parameters, the urban sustainability is divided into 5 levels (see Figure 9(b)). However, we found that 3 and 4 are shared green spaces that are excluded in the evaluation system of sustainable communities. Therefore, on a datadriven basis, we combine value judgments to obtain a sustainable urban community development rating, which is divided into three categories: 0 (low), 1 (medium), and 2 (high).

The spatial features of urban sustainability levels are shown in Figure 9(a). The visualization of principal components with sustainability levels is illustrated in Figure 9(b). Urban sustainability levels are significantly positively correlated with PC1. As PC1 increases, the sustainability level rises. At the same time, urban sustainability shows a negative correlation with PC2. According to the landscape implications of PC1, community land use types with a high level of sustainability are rich and dispersed. The significant synergy between the two indicates that the landscape is an important factor in determining the community sustainability level. Figure 9(c) indicates that the transition from low sustainable to medium sustainable communities is not only an alteration in landscape configuration but also a dramatic change in ESV. Low sustainability level community is dominated by IH and SB with low ESV. Communities of high sustainability levels are V-dominated and accompanied by a high level of landscape sustainability and ESV. The results found that different sustainability levels are dominated by different factors and show significant spatial distribution. In distribution spatial of ESV and landscape configuration in combination with the above housing, most of 253 communities of high sustainability level are located in rivers and emerging urban areas, accompanied by high landscape sustainability. Compared with lower-level communities, 276 communities of the medium sustainability level located in areas with higher ecosystem services, and 127 communities of low sustainability level are mainly distributed in older urban areas with poor housing such as Yuexiu.

Sustainable evaluation through a data-driven approach that does not require much intervention from human parameters is particularly important for objective evaluation, and research must make a clear distinction between sustainable and unsustainable development and not be confused in policy formulation [55]. Simultaneously, existing cities worldwide are aging and much in need of infrastructure replacement [56].

\section{Discussion}

Sustainability assessments are increasingly regarded as an important tool to help transform sustainable urban ecosystems [57]. Nowadays, ecological protection and urbanization seem to be two contradictory concerns. But in fact, rapid urbanization does not mean to say that at the expense of ecosystem damage, policymakers should regard urban development and ecological benefits as mutually reinforcing drivers of sustainable urban development [58]. Each city needs to find its sustainable development path on the way forward. Besides, the connotation of urban sustainability is not confined to ecology, but also economic and social levels. 


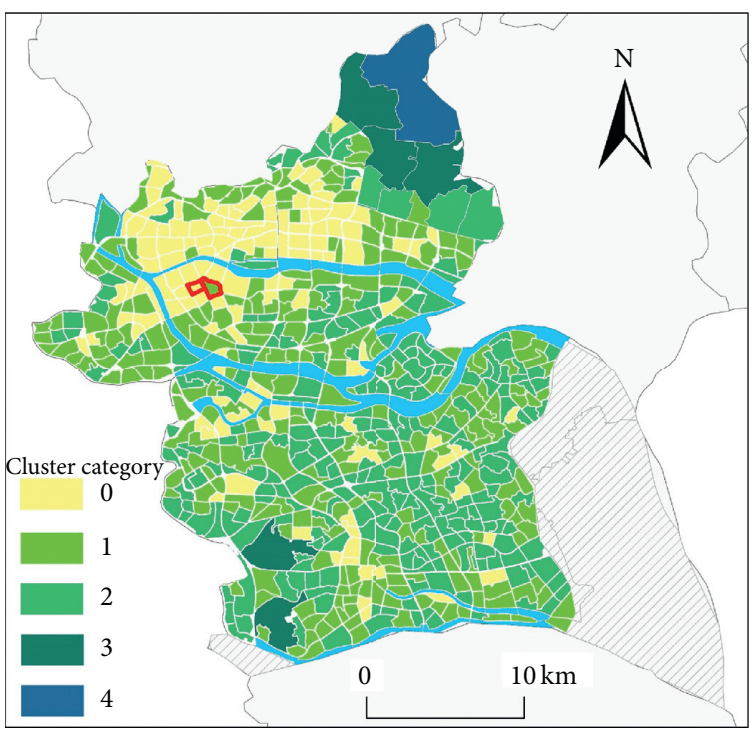

(a)

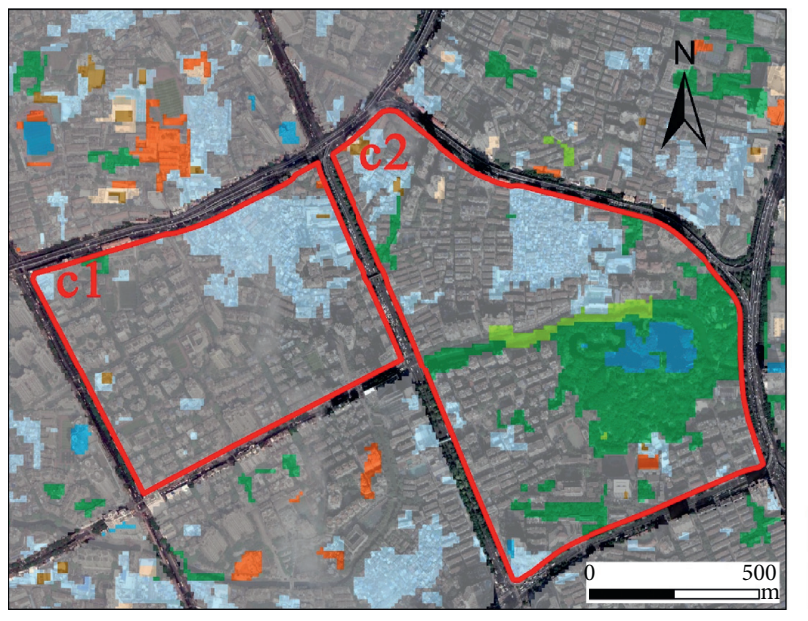

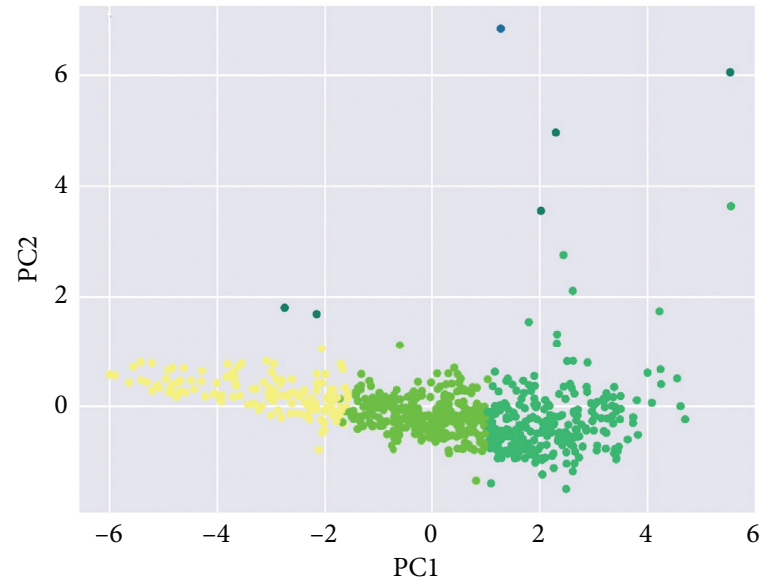

Sustainability level

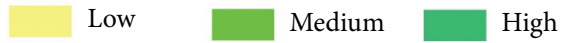

(b)

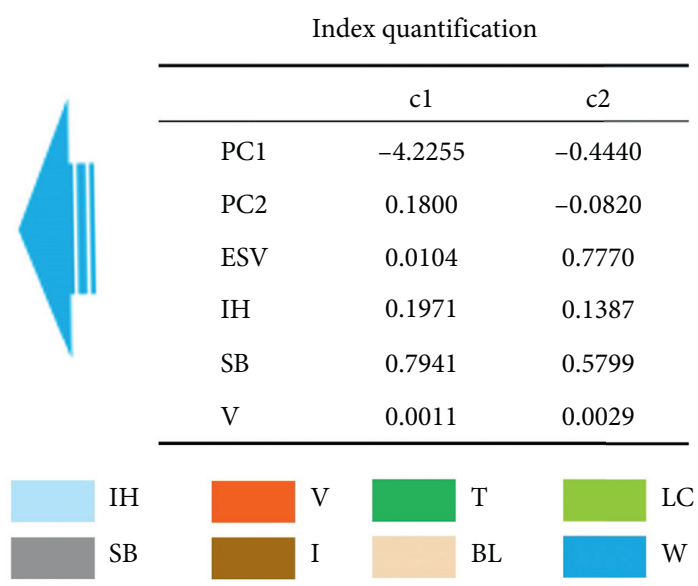

(c)

FIGURE 9: Urban sustainability. (a) Sustainability in the traffic community. (b) Visualization of principal components. (c) Examples of differences between low-level sustainability and high-level sustainability.

It is pointed out that different economic and social levels have different abilities to transform nature, resulting in different land cover and land use. Therefore, we can retrieve the land use information to obtain the corresponding economic and social levels, and combine land cover to present ecology and environments. There are two considerations to note regarding the risk $R$ of land use and land cover classification. The first situations: when a common machine learning algorithm is difficult to classify RS mixed pixels well, $R$ represents a risk. The second situation is as follows: when the state-of-the-art algorithm classifies correctly, $R$ represents a ground complexity, such as cartographic-generalization, and it can summarize and merge features. In this study, the classification of housing, which cannot accurately classify, using Sentinel $10 \mathrm{~m}$ resolution exists mixed pixels [59]. This place $R$ can be considered as a risk assessment indicator. This paper takes full advantage of the timeliness of remotely sensed data, which can be extended to other regions or countries where other statistics are lacking. Socioeconomic disparities usually shape different land use schemes.

The scheme design of land use classification is of great application value to the dynamic monitoring of urban sustainability, which can highlight the contradictions in urban development, explore distinct urban characteristics [60], and complement the global sustainable urban evaluation framework. We provide a sustainable solution to be a viable route based on EOS. At the same time, the timeliness and spatial resolution of EOS possess incomparable advantages over statistical data, and the value of the method in urban sustainability is gradually being paid attention to and adopted by researchers.

In future research, multiple source spatial data (e.g., point of interest, location-based service, and trajectory data) can be used to classify urban land use, and more land use attributes can be added to ensure urban sustainable 
evaluation on the premise of ensuring spatial resolution of RS data, and we can further analyze sustainability from the perspective of urban vitality based on open big data (e.g., Dianping and Baidu heat map data). Simultaneously, quantitative sustainable indicators can be established directly in the context of integrated ecosystem services and landscape sustainability. We need to advance the ecosystem services and human well-being of our cities by taking advantage of the enormous potential offered by data science and technology.

\section{Conclusion}

Although various definitions of urban community sustainability exist, they all focus on long-term development, which reflected in housing, ecosystem services, and landscape. This study evaluates the sustainability of 702 communities in a data-driven manner through quantitating the interrelationship among housing, ecosystem services, and landscape configuration. The main conclusions are as follows:

(1) The synergy effects of housing, ecosystem services, and landscape configuration are significant, among which landscape configuration has the greatest impact on sustainability. Housing is highly related to ecosystem services. Especially, informal housing proportion is negatively related to ESV, which reflects the contradiction between ecological conditions and human housing well-being.

(2) The community sustainable level presents significant spatial heterogeneity. Most of 253 communities of high sustainability level are located in rivers and emerging urban areas, accompanied by high landscape sustainability, while 276 communities with medium sustainability level are located in areas with high ecosystem services and 127 communities with low sustainability level are mainly distributed in older urban areas with poor housing such as Yuexiu.

(3) The negative synergy between the older city and sustainability unveils the hidden dangers of early urban planning in China. Compared with the new urban areas, the older urban areas are less sustainable. Urban renewal and sustainable transformation plans should be different among communities with different development levels.

A data-driven sustainable framework that combines housing, landscape configuration, and ecosystem services can quantitatively evaluate the sustainability level of the community and provide a specific and effective data-based reference for urban renewal. The transition towards a sustainable community should be based on scientific urban planning accompanied by sustainable landscape configuration, and for the transition of communities of low sustainability level, priority should be given to housing reconstruction and then improving ecological conditions.

\section{Data Availability}

The experiment is based on the GEE platform, in which data preprocessing, sample selection and classification, NPP spatiotemporal adjustment factor calculation implementation code, and the repository address of all codes are as follows: https://code.earthengine.google.com/b4f834d9b2676d529346c 6206566ef3b, https://code.earthengine.google.com/7f5aacffbb5 8ebcb6bf4d78658dd6119, https://code.earthengine.google.com /ea6180387fc0d6e5b1ffla63862288e2, and https:/github.com/ HaoweiGis/Assessing-Urban-Sustainability.

\section{Conflicts of Interest}

The authors declare that there are no conflicts of interest regarding the publication of this paper.

\section{Acknowledgments}

This research was funded by the Strategic Priority Research Program of the Chinese Academy of Sciences (grant no. XDA19040502), National Natural Science Foundation of China (grant nos. 41701173 and 41961027), Science Foundation for the Excellent Youth Scholars of Ministry of Education of China (grant no. 17YJCZH268), and LZJTU EP (grant no. 201806).

\section{References}

[1] S. Parnell, "Defining a global urban development agenda," World Development, vol. 78, pp. 529-540, 2016.

[2] M. Acuto, "Global science for city policy," Science, vol. 359, no. 6372, pp. 165-166, 2018.

[3] D. Wachsmuth, D. A. Cohen, and H. Angelo, "Expand the frontiers of urban sustainability," Nature, vol. 536, no. 7617, pp. 391-393, 2016.

[4] K. C. Seto, J. S. Golden, M. Alberti, and B. L. Turner, "Sustainability in an urbanizing planet," Proceedings of the $\mathrm{Na}$ tional Academy of Sciences, vol. 114, no. 34, pp. 8935-8938, 2017.

[5] M. Acuto, S. Parnell, and K. C. Seto, "Building a global urban science," Nature Sustainability, vol. 1, no. 1, pp. 2-4, 2018.

[6] T. Elmqvist, E. Andersson, N. Frantzeskaki et al., "Sustainability and resilience for transformation in the urban century," Nature Sustainability, vol. 2, no. 4, pp. 267-273, 2019.

[7] R. C. Castaneda, The Quest for Sustainable Communities: The Role of Performance Monitoring in the Planning, Design, and Certification of Sustainable Neighborhoods, The University of Texas at San Antonio, San Antonio, TX, USA, 2013.

[8] K. W. Lin and C. M. Shih, "The comparative analysis of neighborhood sustainability assessment tool," Environment and Planning B: Urban Analytics and City Science, vol. 45, no. 1, pp. 90-105, 2018.

[9] L. Huang, L. Yan, and J. Wu, "Assessing urban sustainability of Chinese megacities: 35 years after the economic reform and open-door policy," Landscape and Urban Planning, vol. 145, pp. 57-70, 2016.

[10] Y. Tan, H. Xu, and X. Zhang, "Sustainable urbanization in China: a comprehensive literature review," Cities, vol. 55, pp. 82-93, 2016.

[11] X. Zhang, Y. Wu, and L. Shen, "Embedding "green" in project-based organizations: the way ahead in the 
construction industry?," Journal of Cleaner Production, vol. 107, pp. 420-427, 2015.

[12] L. Cheng, Y. Bae, and W. T. Horton, "A system-level approach for designing multi-family sustainable and energy-efficient housing communities," Sustainable Cities and Society, vol. 44, pp. 183-194, 2019.

[13] I. Bouzguenda, C. Alalouch, and N. Fava, "Towards smart sustainable cities: a review of the role digital citizen participation could play in advancing social sustainability," Sustainable Cities and Society, vol. 50, Article ID 101627, 2019.

[14] Q. Zhang, S. Liu, T. Wang et al., "Urbanization impacts on greenhouse gas (GHG) emissions of the water infrastructure in China: trade-offs among sustainable development goals (SDGs)," Journal of Cleaner Production, vol. 232, pp. 474-486, 2019.

[15] V. Akuraju, P. Pradhan, D. Haase, J. P. Kropp, and D. Rybski, "Relating SDG11 indicators and urban scaling - an exploratory study," Sustainable Cities and Society, vol. 52, Article ID 101853, 2020.

[16] F. Ali-Toudert and L. Ji, "Modeling and measuring urban sustainability in multi-criteria based systems - a challenging issue," Ecological Indicators, vol. 73, pp. 597-611, 2017.

[17] F. Ruan, L. Yan, and D. Wang, "The complexity for the resource-based cities in China on creating sustainable development," Cities, vol. 97, p. 102571, 2020.

[18] R. V. O'neill, J. R. Krummel, R. H. Gardner et al., "Indices of landscape pattern," Landscape Ecology, vol. 1, no. 3, pp. 153-162, 1988.

[19] L. Zhilin, X. Gong, J. Chen et al., "Functional requirements of systems for visualization of sustainable development goal (SDG) indicators," Journal of Geovisualization and Spatial Analysis, vol. 4, no. 1, pp. 1-10, 2020.

[20] S. Pincetl, "Nature, urban development and sustainabilitywhat new elements are needed for a more comprehensive understanding?," Cities, vol. 29, pp. S32-S37, 2012.

[21] T. Dong, L. Jiao, G. Xu, L. Yang, and J. Liu, "Towards sustainability? analyzing changing urban form patterns in the United States, Europe, and China," Science of The Total Environment, vol. 671, pp. 632-643, 2019.

[22] J. Wu, "Urban ecology and sustainability: the state-of-thescience and future directions," Landscape and Urban Planning, vol. 125, pp. 209-221, 2014.

[23] A. Morris, J. Zuo, Y. Wang, and J. Wang, "Readiness for sustainable community: a case study of Green Star Communities," Journal of Cleaner Production, vol. 173, pp. 308317, 2018.

[24] D. R. Godschalk, "Land use planning challenges: coping with conflicts in visions of sustainable development and livable communities," Journal of the American Planning Association, vol. 70, no. 1, pp. 5-13, 2004.

[25] J. Montoya, I. Cartes, and A. Zumelzu, "Indicators for evaluating sustainability in Bogota's informal settlements: definition and validation," Sustainable Cities and Society, vol. 53, p. 101896, 2020.

[26] R. F. M. Ameen and M. Mourshed, "Urban sustainability assessment framework development: the ranking and weighting of sustainability indicators using analytic hierarchy process," Sustainable Cities and Society, vol. 44, pp. 356-366, 2019.

[27] T. Lützkendorf and M. Balouktsi, “Assessing a sustainable urban development: typology of indicators and sources of information," Procedia Environmental Sciences, vol. 38, pp. 546-553, 2017.
[28] V. K. Turner and D. H. Kaplan, "Geographic perspectives on urban sustainability: past, current, and future research trajectories," Urban Geography, vol. 40, no. 3, pp. 267-278, 2019.

[29] W. C. Jochem, T. J. Bird, and A. J. Tatem, "Identifying residential neighbourhood types from settlement points in a machine learning approach," Computers, Environment and Urban Systems, vol. 69, pp. 104-113, 2018.

[30] A. Guo, J. Yang, X. Xiao, J. Xia, C. Jin, and X. Li, "Influences of urban spatial form on urban heat island effects at the community level in China," Sustainable Cities and Society, vol. 53, Article ID 101972, 2020.

[31] J. Yang, X. Luo, C. Jin, X. Xiao, and J. Xia, "Spatiotemporal patterns of vegetation phenology along the urban-rural gradient in Coastal Dalian, China," Urban Forestry \& Urban Greening, vol. 54, Article ID 126784, 2020.

[32] A. Frick and S. Tervooren, "A framework for the long-term monitoring of urban green volume based on multi-temporal and multi-sensoral remote sensing data," Journal of Geovisualization and Spatial Analysis, vol. 3, no. 1, p. 6, 2019.

[33] Y. Ju, S. Lindbergh, Y. He, and J. D. Radke, "Climate-related uncertainties in urban exposure to sea level rise and storm surge flooding: a multi-temporal and multi-scenario analysis," Cities, vol. 92, pp. 230-246, 2019.

[34] Y. Yao, C. Qian, Y. Hong et al., "Delineating mixed urban "jobs-housing" patterns at a fine scale by using high spatial resolution remote-sensing imagery," Complexity, vol. 2020, Article ID 8018629, 13 pages, 2020.

[35] Y. Wang, X. Li, J. Li, Z. Huang, and R. Xiao, "Impact of rapid urbanization on vulnerability of land system from complex networks view: a methodological approach," Complexity, vol. 2018, Article ID 8561675, 18 pages, 2018.

[36] T. Yu, G. Q. Shen, Q. Shi, H. W. Zheng, G. Wang, and K. Xu, "Evaluating social sustainability of urban housing demolition in Shanghai, China," Journal of Cleaner Production, vol. 153, pp. 26-40, 2017.

[37] S. Han and C. Hao, "The essence of "multiple plans integration" and planning compilation," Planners, vol. 31, no. 2, pp. 57-62, 2015.

[38] W. Y. Chen, "Environmental externalities of urban river pollution and restoration: a hedonic analysis in Guangzhou (China)," Landscape and Urban Planning, vol. 157, pp. 170179, 2017.

[39] J. Gong, Z. Hu, W. Chen, Y. Liu, and J. Wang, "Urban expansion dynamics and modes in metropolitan Guangzhou, China," Land Use Policy, vol. 72, pp. 100-109, 2018.

[40] C. Cortes and V. Vapnik, "Support-vector networks," Machine Learning, vol. 20, no. 3, pp. 273-297, 1995.

[41] L. Breiman, "Random forests," Machine Learning, vol. 45, no. 1, pp. 5-32, 2001.

[42] C. Pelletier, S. Valero, J. Inglada, N. Champion, and G. Dedieu, "Assessing the robustness of Random Forests to map land cover with high resolution satellite image time series over large areas," Remote Sensing of Environment, vol. 187, pp. 156-168, 2016

[43] L. H. Nguyen, D. R. Joshi, D. E. Clay, and G. M. Henebry, "Characterizing land cover/land use from multiple years of Landsat and MODIS time series: a novel approach using land surface phenology modeling and random forest classifier," Remote Sensing of Environment, vol. 238, Article ID 111017, 2018.

[44] S. Liu, Z. Qi, X. Li, and A. Yeh, "Integration of convolutional neural networks and object-based post-classification refinement for land use and land cover mapping with optical and SAR data," Remote Sensing, vol. 11, no. 6, p. 690, 2019. 
[45] R. Costanza, R. d'Arge, R. de Groot et al., "The value of the world's ecosystem services and natural capital," Nature, vol. 387, no. 6630, pp. 253-260, 1997.

[46] X. Wang, X. Zhang, X. Feng, S. Liu, L. Yin, and Y. Chen, "Trade-offs and synergies of ecosystem services in karst area of China driven by grain-for-green Program," Chinese Geographical Science, vol. 30, no. 1, pp. 101-114, 2020.

[47] G. Xe, C. Zhang, L. Zhang, W. Chen, and S. Li, "Improvement of the evaluation method for ecosystem service value based on per unit area," Journal of Nature Resources, vol. 30, no. 8, pp. 1243-1254, 2015.

[48] S. W. Running and M. Zhao, "Daily GPP and annual NPP (MOD17A2/A3) products NASA Earth Observing System MODIS land algorithm," MOD17 User's Guide, vol. 2015, 2015.

[49] J. B. Dunning, B. J. Danielson, and H. R. Pulliam, "Ecological processes that affect populations in complex landscapes," Oikos, vol. 65, no. 1, pp. 169-175, 1992.

[50] A. Mascarenhas, L. M. Nunes, and T. B. Ramos, "Selection of sustainability indicators for planning: combining stakeholders' participation and data reduction techniques," Journal of Cleaner Production, vol. 92, pp. 295-307, 2015.

[51] M. J. McDonnell and I. MacGregor-Fors, "The ecological future of cities," Science, vol. 352, no. 6288, pp. 936-938, 2016.

[52] J. Liang, K. Hu, and T. Dai, "Ecological network analysis quantifying the sustainability of regional economies: a case study of Guangdong province in China," Chinese Geographical Science, vol. 28, no. 1, pp. 127-136, 2018.

[53] D. Hope, C. Gries, W. Zhu et al., "Socioeconomics drive urban plant diversity," Proceedings of the National Academy of Sciences, vol. 100, no. 15, pp. 8788-8792, 2003.

[54] J. Wu: "Urban Sustainability: An Inevitable Goal of Landscape Research," Springer, Berlin, Germany, 2010.

[55] P. Verma and A. S. Raghubanshi, "Urban sustainability indicators: challenges and opportunities," Ecological Indicators, vol. 93, pp. 282-291, 2018.

[56] A. Ramaswami, A. G. Russell, P. J. Culligan, K. R. Sharma, and E. Kumar, "Meta-principles for developing smart, sustainable, and healthy cities," Science, vol. 352, no. 6288, pp. 940-943, 2016.

[57] D. Dizdaroglu, "Developing micro-level urban ecosystem indicators for sustainability assessment," Environmental Impact Assessment Review, vol. 54, pp. 119-124, 2015.

[58] J. Yang, Y. Guan, J. Xia, C. Jin, and X. Li, "Spatiotemporal variation characteristics of green space ecosystem service value at urban fringes: a case study on Ganjingzi District in Dalian, China," Science of the Total Environment, vol. 639, pp. 1453-1461, 2018.

[59] Z. Hongwei et al., "A synthesizing land-cover classification method based on Google earth engine: a case study in nzhelele and levhuvu catchments, South Africa," Chinese Geographical Science, vol. 30, no. 3, p. 397, 2020.

[60] I. Walde, S. Hese, C. Berger, and C. Schmullius, "From land cover-graphs to urban structure types," International Journal of Geographical Information Science, vol. 28, no. 3, pp. 584609, 2014. 